\title{
Toxiphobia conditioning with exteroceptive cues
}

\author{
RICHARD V. KRANE \\ Acadia University, Wolfville, Nova Scotia BOP IXO, Canada
}

\begin{abstract}
The types of conditioned properties acquired by novel (i.e., nonpreexposed) or familiar (i.e., preexposed) exteroceptive cues that were paired with toxicosis, in the absence of a flavor CS, were evaluated in four experiments. In Experiment 1, the conditioned properties of novel exteroceptive cues served to block the acquisition of an aversion to a flavor CS during flavor conditioning and to suppress the ingestion response during flavor testing; animals failed to suppress their ingestion of either the flavor CS or a neutral flavor when tested in the absence of the exteroceptive CS, but suppressed their ingestion of both the flavor CS and the neutral flavor when tested in the presence of the exteroceptive CS. In Experiment 2, preexposed exteroceptive cues that had been paired with toxicosis failed to provide evidence of such conditioned properties. Experiment 3 demonstrated that preexposed contextual cues that were reinforced in compound with novel exteroceptive cues failed to acquire the conditioned properties acquired by nonpreexposed contextual cues under the same conditions of reinforcement. Finally, in Experiment 4, the conditioned properties of the novel exteroceptive cues served to evoke a conditioned gastrointestinal response that gradually extinguished as a function of repeated nonreinforced exposures to the exteroceptive cues, and, in the absence of such extinction, the conditioned properties served to block the acquisition of a flavor aversion.
\end{abstract}

In contrast to previous reports of failures to obtain evidence of conditioning when exteroceptive CSs are paired with interoceptive USs (Garcia \& Koelling, 1966, 1967; Garcia, McGowan, Ervin, \& Koelling, 1968), more recent research has shown that relatively novel exteroceptive cues will acquire aversive properties when paired with an interoceptive US (Best, Best, \& Mickley, 1973; Mitchell, Kirschbaum, \& Perry, 1975; Revusky \& Parker, 1976; Rozin, 1969, Experiment 3). However, the fact that exteroceptive cues have been found to acquire relatively little aversiveness if the CS-US interval is a matter of hours (Revusky \& Garcia, 1970; Revusky \& Parker, 1976) or if the exteroceptive cue is presented in compound with a flavor cue (Best et al., 1973; Revusky \& Parker, 1976) might be interpreted as providing support for a weak form of what Bitterman $(1975,1976)$ has termed the "associative predisposition" hypothesis: although animals readily associate exteroceptive cues with an interoceptive US, they are even more prepared to associate interoceptive cues with an interoceptive US. But proponents of general-process learning theory (e.g., Krane \& Wagner, 1975) might argue that such findings simply reflect differences in the qualitative properties of exteroceptive and interoceptive cues (e.g., differences in trace persistence, susceptibility to interference, relative novelty, etc.), rather than differential associative predispositions.

This research was supported by Grant A9796 from the National Research Council of Canada. The assistance of James Ransom in data collection is gratefully acknowledged. Requests for reprints should be addressed to Richard V. Krane, Department of Psychology, Acadia University, Wolfville, Nova Scotia BOP 1X0, Canada.
Of particular relevance here is Holland's (1977) research. Holland found that very different forms of conditioned responses may develop to qualitatively different CSs (i.e., auditory vs. visual) that are reinforced with the same US. This finding suggests that differences obtained with some particular measure of conditioned responding might not reflect differences in associative strengths but rather differences in "CS-CR compatibility" that are unrelated to associative strength; if so, associative strength might be indexed better by some other means. The results of a number of recent experiments indicate that a measure of the ability of one stimulus, $\mathrm{CS}_{\mathrm{A}}$, to block the development of a conditioned response to another stimulus, $\mathrm{CS}_{\mathrm{B}}$, or to serve as a second-order reinforcer for $\mathrm{CS}_{\mathrm{B}}$, may provide a better index of the associative strength of $\mathrm{CS}_{\mathrm{A}}$ than a measure of the ability of $\mathrm{CS}_{\mathrm{A}}$ to itself evoke a particular conditioned response (e.g., Blanchard \& Honig, 1976; Holland, 1977; Holland \& Rescorla, 1975a, 1975b; Rescorla, 1973; Rescorla \& Furrow, 1977; Rudy, Iwens, \& Best, 1977). In the Holland (1977) study, for example, two qualitatively different $\mathrm{CSs}, \mathrm{CS}_{\mathrm{A}}$ and $\mathrm{CS}_{\mathrm{B}}$, were each separately reinforced with a food US, and certain conditioned responses were observed to develop to $\mathrm{CS}_{\mathrm{A}}$ but not to $\mathrm{CS}_{\mathrm{B}}$. Although it appeared that $\mathrm{CS}_{\mathrm{B}}$ had not been associated with the food US, Holland demonstrated that when $\mathrm{CS}_{\mathrm{B}}$ was subsequently placed in compound with a neutral $\mathrm{CS}_{\mathrm{A}}$ and the compound was reinforced with the food US, $\mathrm{CS}_{\mathrm{B}}$ blocked the acquisition of conditioned responding to $\mathrm{CS}_{\mathrm{A}}$.

In view of Holland's (1977) research, it is plausible to suggest that studies which have found exteroceptive 
cues to acquire relatively little aversiveness when reinforced in compound with a flavor cue may not have employed an optimal measure of associative strength. A measure of the ability of an exteroceptive cue to block the development of an aversion to a flavor cue might provide a more adequate assessment of the extent to which animals will associate an exteroceptive CS with an interoceptive US. Thus, the purpose of the present research was to attempt to evaluate the conditioned properties acquired by novel (i.e., nonpreexposed) or familiar (i.e., preexposed) exteroceptive cues that were reinforced with toxicosis, in the absence of a flavor cue, and then presented in compound with a flavor cue and reinforced with toxicosis.

\section{EXPERIMENT 1}

Rudy, Iwens, and Best (1977) recently reported a series of experiments in which the pairing of novel exteroceptive cues and toxicosis was found to attenuate the subsequent development of an aversion to a flavor cue, regardless of whether the previously reinforced novel cues were or were not present during flavor conditioning. The authors postulated that pairing the novel exteroceptive cues with toxicosis resulted in the preexposed contextual cues becoming associated with the toxicosis. As a consequence, the contextual cues signaled the occurrence of toxicosis during flavor conditioning and thus blocked the formation of an association between the flavor cue and the toxicosis. Unfortunately, Rudy et al. did not attempt to determine whether the exteroceptive cues had acquired aversive properties that would also cause the animals to suppress ingestion of non-CS fluids when tested in the presence of such cues. In addition to providing independent evidence of the animals having associated the preexposed contextual cues, rather than the novel cues, with the toxicosis, such a demonstration would have provided information concerning the relative sensitivity of two different measures of associative strength. The purpose of the present experiment was to obtain such information.

Two groups of animals were preexposed to exteroceptive cues $(C)$ and then poisoned either in the presence of these cues (Group CP) or in the presence of novel exteroceptive cues (Group BP) (see Table 1). Following these "context conditioning" trials, both groups of animals were presented with a novel flavor cue $(\mathrm{X})$ in compound with the novel exteroceptive cues and again were poisoned. The conditioned properties acquired by the preexposed and novel exteroceptive cues were assessed by measuring ingestion of the flavor CS (X), as well as ingestion of a neutral flavor $(Z)$, in the presence of the preexposed $(C)$ or the novel (B) exteroceptive cues.

Consistent with the literature on blocking, it was assumed that during context conditioning Group BP would associate the novel exteroceptive cues with the toxicosis and that this would result in blocking of the flavor aversion during the subsequent reinforcement of flavor $\mathrm{X}$ in compound with the novel exteroceptive cues. Thus, it was anticipated that Group BP would not suppress their ingestion of either flavor $\mathrm{X}$ or the neutral flavor $\mathrm{Z}$ during posttests conducted in the presence of the preexposed exteroceptive cues $(C)$. However, assuming that the novel exteroceptive cues acquired aversive properties as a consequence of having been associated with toxicosis, these same animals were expected to suppress their ingestion of both flavor $\mathrm{X}$ and the neutral flavor $\mathrm{Z}$ during posttests conducted in the presence of the novel exteroceptive cues (B).

Table 1

Design of Experiments 14

\begin{tabular}{|c|c|c|c|c|}
\hline & $\begin{array}{c}\text { Cue } \\
\text { Preexposure }\end{array}$ & $\begin{array}{c}\text { Context } \\
\text { Conditioning }\end{array}$ & $\begin{array}{c}\text { Flavor } \\
\text { Conditioning }\end{array}$ & $\begin{array}{c}\text { Test } \\
\text { Condition }\end{array}$ \\
\hline & \multicolumn{4}{|c|}{ Experiment 1} \\
\hline Group BP/BXP & $\mathrm{C}$ & BP & BXP & $\mathrm{BX} \mathrm{BZ} \mathrm{CX} \mathrm{CZ}$ \\
\hline \multirow[t]{2}{*}{ Group CP/BXP } & $\mathrm{C}$ & $\mathrm{CP}$ & BXP & $\mathrm{BX} \mathrm{BZ} \mathrm{CX} \mathrm{CZ}$ \\
\hline & \multicolumn{4}{|c|}{ Experiment 2} \\
\hline Group BP/CXP & $\mathrm{C}$ & BP & CXP & BX BZ CX CZ \\
\hline \multirow[t]{2}{*}{ Group CP/CXP } & $\mathrm{C}$ & $\mathrm{CP}$ & CXP & $\mathrm{BX} \mathrm{BZ} \mathrm{CX} \mathrm{CZ}$ \\
\hline & \multicolumn{4}{|c|}{ Experiment 3} \\
\hline Group BCP/BCXP & none & BCP & BCXP & NX CX BX \\
\hline Group C/BCP/BCXP & $\mathrm{C}$ & BCP & $\mathrm{BCXP}$ & $\mathrm{NX} C X \mathrm{BX}$ \\
\hline Group BCP/CXP & none & BCP & CXP & NX CX BX \\
\hline Group C/BCP/CXP & $\mathrm{C}$ & $\mathrm{BCP}$ & CXP & NX CX BX \\
\hline \multirow[t]{2}{*}{ Group $\mathrm{C} / \mathrm{CP} / \mathrm{CXP}$} & $\mathrm{C}$ & $\mathrm{CP}$ & CXP & NX CX BX \\
\hline & \multicolumn{4}{|c|}{ Experiment 4} \\
\hline Group BP/B/BXP & none & BP (B) & BXP & NX \\
\hline Group BP/rest/BXP & none & $\mathrm{BP}$ (rest) & BXP & NX \\
\hline
\end{tabular}

Note-B=Environment $B, C=$ Environment $C, N=$ neutral environment $X=$ Flavor $C S, Z=$ neutral flavor, $P=$ poison. 
The literature on CS preexposure effects (cf. Lubow, 1973) also led us to anticipate that the preexposed exteroceptive cues would not acquire conditioned properties during context conditioning for animals of Group CP. Moreover, reinforcement of flavor $\mathrm{X}$ in compound with the novel exteroceptive cues was not expected to block acquisition of a flavor aversion in these animals because the novel cues had not been paired with toxicosis prior to flavor conditioning. Thus, it was further predicted that Group CP would not suppress their ingestion of the neutral flavor $\mathrm{Z}$ during posttests conducted in the presence of either the preexposed $(C)$ or the novel (B) exteroceptive cues, but that they would suppress their ingestion of flavor $\mathrm{X}$ in the presence of both the preexposed cues and the novel cues.

\section{Method}

Subjects and Apparatus. The subjects were 24 male albino Sprague-Dawley rats $(350-450 \mathrm{~g})$, obtained from the Charles River Breeding Laboratories, Wilmington, Massachusetts. Upon arrival at the laboratory, the rats were housed in the vivarium in individual cages, provided with free access to food and water for approximately 1 month, and then placed on a 23.8 -h water deprivation schedule.

The apparatus consisted of calibrated drinking tubes, a set of eight standard size $(25 \times 20 \times 18 \mathrm{~cm})$ rat cages (Wahman) and a set of eight enclosed "holding boxes" $(26 \times 15 \times 18 \mathrm{~cm})$, all of which were located in a quiet isolation room that had much lower levels of ambient illumination and sound than did the vivarium. The top and sides of each holding box were wood, while the floor was a piece of black acrylic that lay on top of a piece of wire mesh. The relatively novel set of exteroceptive stimuli to which animals were exposed when placed in the dark, enclosed boxes in the isolation room constituted environment $B(B)$, and the more familiar set of exteroceptive stimuli to which animals were exposed when placed in the standard rat cages in the isolation room constituted environment $\mathrm{C}(\mathrm{C})$.

Tests of conditioning involved the use of two flavors, the flavor CS (a .24\% sodium saccharin solution) and a "neutral" test flavor (a .9\% saline solution). To simplify labeling, the terms flavor $\mathrm{X}$ and flavor $\mathrm{Z}$ are used to refer to the flavor $\mathrm{CS}$ and the neutral test flavor, respectively.

Preliminary training. Three weeks before the experiment proper was begun, all animals were placed on a water-deprivation schedule that permitted them to drink water in their home cages in the vivarium for 7 min each day. During Days 1-3 of the experiment, all animals were allowed to ingest three different novel flavored solutions (butterscotch, almond, maple) in their home cages. The intention was to reduce the rats" "neophobia" (Rozin, 1968) for later ingesting a novel flavor in a novel place. Four counterbalanced pretests were administered over Days 4-7, each animal being exposed to $7 \mathrm{~min}$ of flavor $\mathrm{X}$ or flavor $\mathrm{Z}$ in environments $B$ and $C$. The purpose of these pretests was to establish a baseline measure of ingestion of flavors $\mathrm{X}$ and $\mathrm{Z}$ in the two environments.

In order to reduce the novelty of environment $C$ relative to $B$, all animals were placed in $C$ and allowed $7 \mathrm{~min}$ of access to tap water on Days $9,10,13$, and 19 and to a different flavored solution (peppermint-, rum-, orange-, and brandy-flavored solutions) on Days $12,14,16$, and 18 , respectively. On Days 8,11 , $15,17,21,23$, and 25 , the animals ingested the plain or flavored water in their home cages in the vivarium, the flavored solutions being vanilla, grape, coconut, tap water, vinegar, milk, and lemon, respectively. Flavor solutions were prepared by adding .5 to $2.0 \mathrm{ml}$ of commercial (e.g., Schwartz or Durkee's) food extracts (pure or artificial) to a liter of distilled water. The animals were exposed to the different flavored solutions in an attempt to minimize potential overshadowing of the exteroceptive CS by flavor $\mathrm{X}$ during flavor conditioning.

Following preexposure of the 24 animals to environment $C, 12$ animals were assigned randomly to Group BP and 12 to Group CP. [In Table ], the letters preceding the slash indicate the environment ( $\mathrm{B}$ or $\mathrm{C}$ ) in which that group experienced poisoning $(\mathrm{P})$ during context conditioning, and the letters following the slash indicate the environment in which all animals ingested the flavor CS (flavor $\mathrm{X}$ ) and experienced poisoning on the flavor conditioning day.]

Context conditioning. The pairing of the novel (B) or the preexposed (C) complex of exteroceptive cues with poisoning occurred on Days 20 and 22 . No fluids were available during these sessions. The animals in Group BP were placed in B, while the animals in Group CP were placed in C; after $1 \mathrm{~min}$, all animals received a $100-\mathrm{mg} / \mathrm{kg}$ dose of $2 \% \mathrm{LiCl}$ solution $(w / v)$, injected $\mathrm{ip}$, and were reexposed to $\mathrm{B}$ or $\mathrm{C}$, respectively, for $30 \mathrm{~min} ; 5 \mathrm{~h}$ later, they were given $7 \mathrm{~min}$ access to unflavored water in their home cages.

Flavor conditioning. Reinforcement of the flavor CS in compound with the exteroceptive CS occurred on Day 24. The animals in both groups were placed in environment $B$ and permitted to ingest flavor $X$ ( $.24 \%$ saccharin solution) for $7 \mathrm{~min}$; $15 \mathrm{~min}$ following the removal of the drinking tubes, each animal was injected with a $100-\mathrm{mg} / \mathrm{kg}$ dose of $2 \% \mathrm{LiCl}$ solution, was returned to $\mathrm{B}$ for $30 \mathrm{~min}$, and then was returned to the vivarium.

Testing. The extent to which the animals associated environments $\mathrm{B}$ and $\mathrm{C}$ with toxicosis was assesed by means of four counterbalanced, single-bottle tests that permitted each animal to ingest each flavor in each environment over Days 26-29. The order of the four tests was equated across the two groups such that in each daily test session 3 of the 12 animals in each group were tested with flavor $\mathrm{X}$ in $\mathrm{B}, 3$ with flavor $\mathrm{X}$ in $\mathrm{C}, 3$ with flavor $\mathrm{Z}$ in $B$, and 3 with flavor $Z$ in $C$, the same as was done during the pretests.

\section{Results and Discussion}

The results of Experiment 1 are presented in Figure 1. Comparisons (one-tailed t tests with $11 \mathrm{df}$ ) of the pretest vs. posttest performance within each group indicated that Group BP suppressed their ingestion of flavor $\mathrm{X}$, the flavor $\mathrm{CS}$, during the posttests in environment $B(p<.005)$, ? ut not during the posttests in $\mathrm{C}(\mathrm{t}<1)$. These same animals also exhibited a tendency to suppress their ingestion of flavor $\mathrm{Z}$, the neutral flavor, during the posttests in B $(t=1.62, p<.07)$, but not during the posttests in $C$ $(t<1)$. In contrast, Group CP suppressed their ingestion of flavor $\mathrm{X}$ during the posttests in both $\mathrm{B}$ and $C$ (ps $<.025$ and .005 , respectively), but did not suppress ingestion of flavor $\mathrm{Z}$ during the posttests in either B or C (both ts $<1$ ).

The pattern of results revealed by these comparisons suggests that the contextual conditioning that Group BP received in environment B resulted in the association of the exteroceptive cues of $B$ with the interoceptive US of toxicosis. As a consequence, the presence of the exteroceptive cues of B during flavor conditioning served to block acquisition of an aversion to flavor $X$ in Group BP, and the presence of the exteroceptive cues of $B$ during testing served to inhibit the ingestion of both the flavor CS (X) and 


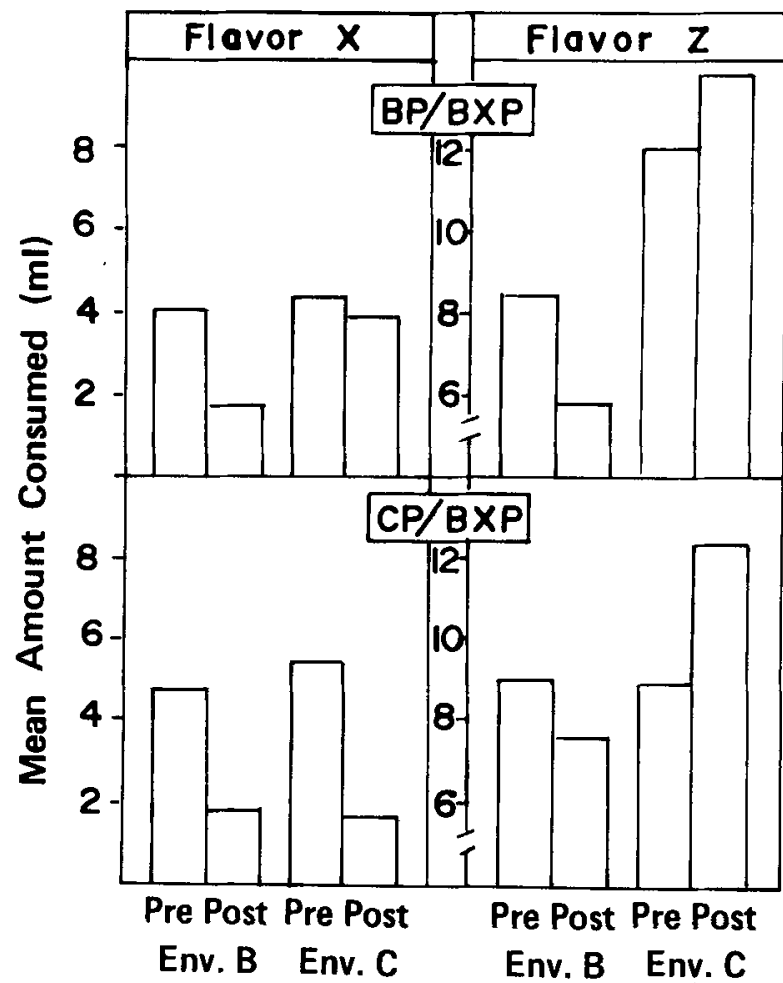

Figure 1. Mean amounts of flavor $X$ (flavor CS) and flavor $Z$ (neutral flavor) ingested in Experiment 1 by Groups BP/BXP and CP/BXP during the pre- and posttests in environments B (exteroceptive CS) and C.

the neutral flavor $(Z)$. That these animals did not inhibit ingestion of either flavor $\mathrm{X}$ or flavor $\mathrm{Z}$ in environment $\mathrm{C}$ is consistent with this interpretation.

The results also suggest that in Group CP neither context conditioning trials that occurred in environment $\mathrm{C}$ nor the flavor conditioning trial in $\mathrm{B}$ resulted in the exteroceptive cues of these environments being associated with toxicosis. The failure to observe a reliable decrease in the ingestion of the neutral flavor $Z$ in $B$ or $C$ supports this suggestion. Thus, it seems reasonable to interpret the decrease in the ingestion of flavor X observed for Group CP in both B and C as reflecting conditioned properties of flavor $\mathrm{X}$ rather than conditioned properties of environments $B$ and C. Although Groups BP and CP both suppressed their ingestion of flavor $\mathrm{X}$ in $\mathrm{B}$, the suppression exhibited by Group BP appears to have been due to an acquired aversiveness of the exteroceptive CS, while the suppression exhibited by Group CP appears to have been due to an acquired aversiveness of the flavor CS.

Especially noteworthy is the relative difference in the extents to which Groups BP and CP suppressed their ingestion of flavor $X$ in $C$. Assume for the moment that the several occasions on which the animals were preexposed to $\mathrm{C}$ resulted in the exteroceptive cues of that environment becoming latently inhibited (Lubow, 1973) and that, as a consequence, the context conditioning trials that Group $\mathrm{CP}$ received in C were, in effect, US preexposure trials. Assume, further, that the context conditioning trials that Group BP received in B resulted in B's acquiring conditioned properties that served to block the acquisition of an aversion to flavor $X$ when flavor $X$ was reinforced in compound with environment $B$. Given these assumptions, it would appear that a nonassociative, US-preexposure procedure interferes much less with the conditioning of a flavor aversion than does an associative, blocking procedure.

\section{EXPERIMENT 2}

The findings of Rudy et al. (1977) suggest that the context conditioning that Group BP received in the relatively novel environment, $B$, may have been a sufficient condition for producing the observed attenuation of the flavor aversion, that is, that the presence of B during flavor conditioning may not have been a necessary condition for producing the attenuation. The purpose of Experiment 2 was to determine whether or not the attenuated aversion observed in Group BP in Experiment 1 would have occurred if the exteroceptive cues of B had not been present during flavor conditioning (Table 1, Group BP/ CXP). A secondary purpose was to determine whether or not an exteroceptive CS to which animals have been preexposed will, when subsequently paired with toxicosis, acquire conditioned properties that serve to block the acquisition of an aversion to a flavor CS or to suppress the ingestion of a neutral flavor (Group CP/CXP).

\section{Method}

The apparatus and general subject population were the same as those employed in Experiment 1. The procedural details of Experiment 2 were identical to those of Experiment 1 in all respects except the following: (1) Environment $C$ was designated as the exteroceptive CS. (2) After preexposing all subjects to $C$, the animals in Group CP $(n=12)$ received context conditioning in $C$, while the animals in Group BP $(n=12)$ received context conditioning in the relatively novel environment B. (3) All animals were then presented with the saccharin CS (flavor $X$ ) in $C$ and poisoned.

\section{Results and Discussion}

Figure 2 shows that, in contrast to the results obtained in Experiment 1, acquisition of an aversion to flavor X was not blocked in Group BP. In accord with the data depicted in the upper panel of Figure 2, one-tailed $t$ tests with $11 \mathrm{df}$ indicated that Group BP strongly suppressed ingestion of flavor $\mathrm{X}$ in both environments $B$ and $C$ (ps $<.005$ ), but did not suppress ingestion of flavor $\mathrm{Z}$ in either $\mathrm{B}$ or $\mathrm{C}(\mathrm{ts}<1)$. The lower panel of Figure 2 shows that Group $C P$ likewise inhibited ingestion of flavor $X$ in both $B$ and $C$ ( $p<.005$ and $p<.05$, respectively), but did not inhibit ingestion of flavor $\mathrm{Z}$ in either environment $\mathrm{B}$ or $\mathrm{C}(\mathrm{ts}<1)$. 


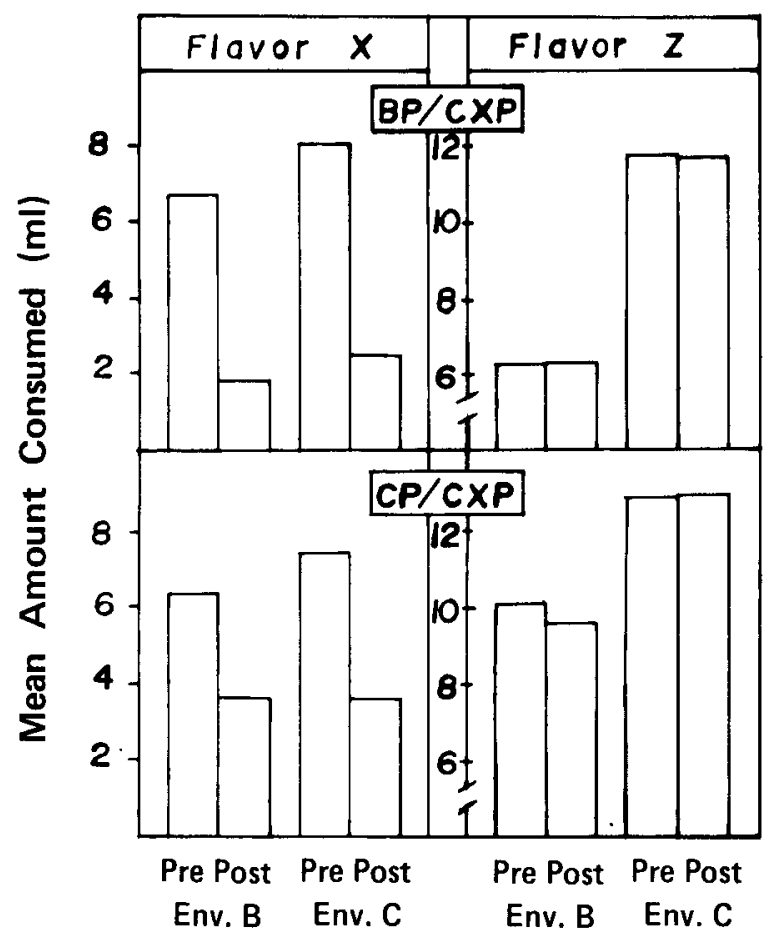

Figure 2. Mean amounts of flavor $X$ (flavor $C S$ ) and flavor $Z$ (neutral flavor) ingested in Experiment 2 by Groups BP/CXP and CP/CXP during the pre- and posttests in environments $B$ and $C$ (exteroceptive CS).

The pattern of results obtained for Group BP suggests that simply exposing animals to novel exteroceptive stimulation (B) prior to the induction of toxicosis was not a sufficient condition for producing an attenuated flavor aversion in the present experiment. Rather than exhibiting an attenuated aversion to flavor X, Group BP suppressed their ingestion of flavor $\mathrm{X}$ in $\mathrm{C}$ as well as in $\mathrm{B}$. Thus, it seems reasonable to conclude that, in Experiment 1, the presence of the exteroceptive cues of $B$ during flavor conditioning was a necessary condition for producing the attenuated flavor aversion observed in Group BP, and that the attenuation represents a true blocking effect.

The pattern of results obtained in Group CP suggests that preexposing animals to $C$ reduced the ability of environment $\mathrm{C}$ to acquire conditioned properties during context conditioning. This, in turn, lowered the likelihood of C's blocking the formation of an association between flavor $\mathrm{X}$ and the toxicosis during flavor conditioning. Unfortunately, the present experiment did not include a comparison condition in which animals were not preexposed to C. Experiment 3 includes such a comparison condition and provides data that are consistent with the above interpretation. Moreover, there is ample evidence in the literature that preexposure to a to-be-conditioned CS can markedly reduce the ability of the CS to acquire conditioned properties (see Lubow, 1973; Rudy et al., 1977, Experiment 4).
In view of the results obtained for Group BP in Experiment 1, the context conditioning that Group BP received in Experiment 2 was expected to result in environment B's acquiring aversive conditioned properties that would serve to suppress the ingestion of both flavor $\mathrm{X}$ and flavor $\mathrm{Z}$ in that environment. But, in Experiment 2, the amount of flavor $Z$ ingested by Group BP in B during the posttest did not differ from the amount ingested during the pretest. The significance of the failure to find a difference is difficult to assess because the unusually small intake of flavor $\mathrm{Z}$ during the pretest in $\mathrm{C}$ (see Figure 2) might have imposed a "floor effect."

There is an obvious limitation to the designs of Experiments 1 and 2. As a consequence of receiving context conditioning in one environment and then flavor conditioning in either that same environment or in a different environment, the two groups of animals necessarily receive different degrees of exposure to the environments in which ingestion of the flavor CS is later tested. Thus, it is possible that the effect of reinforcing a flavor $\mathrm{CS}$ in compound with an exteroceptive CS that has a different history of reinforcement for the two groups of animals is confounded with the effect of measuring ingestion of the flavor CS in a test environment not equally familiar to the two groups. Ideally, ingestion of the flavor CS should be tested in some "neutral" environment, as well as in environments B and C. By this procedure, ingestion of the flavor CS reflects only the degree to which ingestion is controlled by the properties of the flavor CS, and not by the properties of the exteroceptive cues. Experiment 3 employed the "neutral environment" procedure.

\section{EXPERIMENT 3}

In contrast to the failure to find any evidence of blocking in Group BP in Experiment 2, Rudy et al. (1977) observed an attenuation of flavor aversion learning in animals treated much the same as the animals in Group BP were treated in Experiment 2 (Group B-P/S-P in the Rudy et al. study). A possible reason for this discrepancy may be that the animals in the Rudy et al. study experienced the preexposed exteroceptive cues at the same time that they were exposed to the novel exteroceptive cues and poisoned, whereas the animals in Group BP in Experiment 2 did not, during context conditioning, experience the preexposed cues (C) when they were poisoned in the presence of the novel exteroceptive cues (B). The purpose of Experiment 3 was to provide a test of this possibility by employing conditions that more closely resembled the conditions used in the Rudy et al. study.

The basic proposal advanced by Rudy et al. (1977) was that exposure to novel exteroceptive cues just prior to poisoning disrupts the processes responsible 
for latent inhibition. If this assumption is correct, then preexposed exteroceptive cues that are reinforced in compound with novel exteroceptive cues should acquire conditioned properties. As a consequence, subsequent reinforcement of a flavor $C S$ in compound with the preexposed cues should result in blocking of the development of an aversion to the flavor CS.

Rudy et al. (1977) also found that reinforcement of the flavor CS in compound with the preexposed and the novel exteroceptive cues resulted in no more attenuation of the flavor aversion than did reinforcement of the flavor CS in compound with the preexposed exteroceptive cues alone. This finding suggests that, in the Rudy et al. study, the novel exteroceptive cues acquired relatively little associative strength when reinforced in compound with the preexposed exteroceptive cues during the context conditioning phase. However, the pattern of results obtained for Group BP in Experiment 1 of the present study suggests that novel exteroceptive cues that are paired with toxicosis will acquire sufficient associative strength to block the acquisition of a flavor aversion and, at least to some degree, suppress ingestion of a neutral flavor. Thus, a second purpose of Experiment 3 was to determine whether exposure to both the novel exteroceptive cues and the preexposed cues during flavor conditioning would result in greater blocking of the flavor aversion than would exposure to the preexposed cues alone. In view of the fact that Rudy et al. did not attempt to assess the conditioned properties of the novel and preexposed exteroceptive cues by some measure other than blocking, it also was of interest to determine the extent to which animals would inhibit the ingestion response in the presence of such cues.

\section{Method}

Design. The basic experiment employed a 2 by 2 factorial design (see Table 1). Two groups of rats were preexposed, and two groups of rats were not preexposed, to the complex of contextual exteroceptive cues $(C)$ that subsequently was reinforced in compound with a relatively novel, more discrete, set of exteroceptive cues (B) during context conditioning. Following the context conditioning phase, one of the preexposed groups (Group C/BCP/ $\mathrm{CXP}$ ) and one of the nonpreexposed groups (Group BCP/CXP) were poisoned to flavor $X$ (i.e., the flavor $C S$ ) in compound with the contextual cues $(\mathrm{C})$ alone, while the remaining two groups were poisoned to flavor $\mathrm{X}$ in compound with both the novel exteroceptive cues (B) and the contextual cues (Groups C/BCP/ $\mathrm{BCXP}$ and $\mathrm{BCP} / \mathrm{BCXP}$ ). In addition to these four experimental groups, a supplementary control group assessed the effects of pairing novel exteroceptive stimulation with poisoning during the context conditioning phase. Group $\mathrm{C} / \mathrm{CP} / \mathrm{CXP}$ was treated the same as Group C/BCP/CXP except that, during the context conditioning phase, the former group was not exposed to $\mathrm{B}$, but rather was poisoned in the presence of the contextual cues alone. To assess the effects of the experimental manipulations, ingestion of flavor $X$ was first measured in a neutral test environment (i.e., in the absence of either the contextual cues provided by $C$ or the novel exteroceptive cues provided by $B$ ), then, after extinction of the flavor aversion, in the presence of the contextual cues $(C)$ alone, and then in the presence of the novel exteroceptive cues (B).
Subjects and Apparatus. The subjects were 30 experimentally naive male Sprague-Dawley rats $(300-400 \mathrm{~g})$, obtained from the Bio Breeding Laboratories of Canada Ltd., Ottawa, Ontario. Upon arrival at the laboratory, the animals were placed on a 23.6-h water-deprivation schedule that provided them with $20 \mathrm{~min}$ of access to water each day in their home cages.

The apparatus consisted of calibrated drinking tubes, a set of 30 animal cages that differed from the rats' home cages both in size and construction, and a set of eight enclosed "holding boxes" $(26 \times 15 \times 18 \mathrm{~cm})$, all of which were located in a type of observation room that differed in size, illumination, background noise, and temperature from the vivarium in which the animals were housed. In contrast to the vivarium, this room, which was designated as environment $\mathrm{C}(\mathrm{C})$ was smaller and was illuminated by three $60-\mathrm{W}$ red-light bulbs located in a row of ceiling fixtures along the center of the ceiling. A low level of background noise was provided by a dc voltage regulator and a fan-forced electric heater with a thermostat. The temperature of the room was maintained at $25.0^{\circ} \mathrm{C}$, which was $2.5^{\circ} \mathrm{C}$ above that of the vivarium. Because all experimental manipulations were conducted within the confines of this room, the auditory, visual, and thermal stimuli to which the animals were exposed in environment $C$ were viewed as contextual cues that, in many respects, were assumed to be analogous to the contextual cues postulated to have been provided by the experimenter in the Rudy et al. (1977) study.

In contrast to the standard-size $(25 \times 20 \times 18 \mathrm{~cm})$ rat cages (Wahman Mfg. Co.) located in the colony room, the cages located in environment $C$ were larger $(28 \times 22 \times 22 \mathrm{~cm})$ and were constructed entirely of wire grid, thus permitting the animals to be fully exposed to the contextual cues of $\mathrm{C}$. The dark enclosed boxes that provided the novel exteroceptive stimulation were the same as those used in Experiments 1 and 2 and again constituted environment $B$ (B). The boxes were located within environment $C$ in an area adjacent to the cages in which the animals would be exposed to the contextual cues

Phase 1: Preexposure to contextual cues. Following a 3-week water-deprivation period, during which all animals were permitted to drink water in the home cages for $20 \mathrm{~min}$ each day, the animals in Groups $\mathrm{C} / \mathrm{BCP} / \mathrm{CXP}, \mathrm{C} / \mathrm{BCP} / \mathrm{BCXP}$, and $\mathrm{C} / \mathrm{CP} / \mathrm{CXP}$ were exposed to the contextual cues of $\mathrm{C}$ on seven separate occasions. The durations for which the animals were exposed to the cues were $11 / 2 \mathrm{~h}, 10 \mathrm{~min}, 4 \mathrm{~h}$, and $3 / 4 \mathrm{~h}$ on Preexposure Sessions $1-4$, respectively, and $10 \mathrm{~min}$ on Sessions 5-7. On the first preexposure session, two 5-g pellets of Purina Lab Chow were placed in each of the cages in environment $C$, and a drinking tube containing $10 \mathrm{cc}$ of water was attached to the front of each cage. The animals were not provided with water during Sessions 2 and 3 , and at the end of the third session all remaining food was removed. On Sessions 5 and 6 , the animals were provided with $10 \mathrm{cc}$ of water during the last $7 \mathrm{~min}$ of the 10-min exposure period, and on Session 7, water was not made available during the exposure period.

To insure that the nonpreexposed animals received as much handling, exposure to the experimenter, access to water, food, etc., as did the preexposed animals, the animals in Groups $\mathrm{BCP} / \mathrm{CXP}$ and BCP/BCXP were equally often transported to an area of the laboratory in the vicinity of environment $C$ and immediately returned to their home cages, where they were provided with amounts of food and water equivalent to that given to the preexposed animals.

Phase 2: Contextual conditioning. Twenty-four hours following the last preexposure session, the animals in the four experimental groups were transported to environment $C$ and placed, for $3 \mathrm{~min}$, in the dark enclosed boxes that constituted environment $B$. The animals were then removed from the boxes and briefly exposed to the contextual cues of environment $\mathrm{C}$ while being injected with $100 \mathrm{mg} / \mathrm{kg}$ of $\mathrm{LiCl}$; following the injection, they were returned to the boxes for $5 \mathrm{~min}$ and then returned to their home cages in the vivarium. The animals in the control group were simply placed in the cages in environment $\mathrm{C}$, injected with the $\mathrm{LiCl} 3 \mathrm{~min}$ later, returned to the cages for $5 \mathrm{~min}$, and then returned to the vivarium. This same procedure was repeated $24 \mathrm{~h}$ later. 
Phase 3: Flavor conditioning. The day following the second context conditioning trial, all animals were transported to environment $\mathrm{C}$, where Groups $\mathrm{C} / \mathrm{BCP} / \mathrm{CXP}, \mathrm{BCP} / \mathrm{CXP}$, and $\mathrm{C} / \mathrm{CP} / \mathrm{CXP}$ were placed in the cages and exposed only to the contextual cues, while Groups C/BCP/BCXP and BCP/BCXP were placed in the dark boxes and exposed to the novel exteroceptive cues of B. At the end of $3 \mathrm{~min}$, the animals in the latter two groups were removed from the boxes, and a drinking tube containing $5 \mathrm{cc}$ of flavor X (a .12\% sodium saccharin solution, the flavor CS) was attached to each of the cages to which the animals were transferred, as well as to the cages containing the animals in Groups $\mathrm{C} / \mathrm{BCP} / \mathrm{CXP}, \mathrm{BCP} / \mathrm{CXP}$, and $\mathrm{C} / \mathrm{CP} / \mathrm{CXP}$. All animals consumed all $5 \mathrm{cc}$ of flavor $\mathrm{X}$ during the 7 -min period of access to the drinking tube. The animals were then injected with $100 \mathrm{mg} / \mathrm{kg}$ of $\mathrm{LiCl}$, returned to the cages for $5 \mathrm{~min}$, and then returned to the vivarium. Thus, Groups $\mathrm{C} / \mathrm{BCP} / \mathrm{BCXP}$ and $\mathrm{BCP} / \mathrm{BCXP}$ were exposed to flavor $X$ in sequential compound with the novel exteroceptive cues of $\mathrm{B}$ and the contextual cues of $\mathrm{C}$, while Groups $\mathrm{C} / \mathrm{BCP} / \mathrm{CXP}, \mathrm{BCP} / \mathrm{CXP}$, and $\mathrm{C} / \mathrm{CP} / \mathrm{CXP}$ were exposed to flavor $\mathrm{X}$ in compound with the contextual cues alone. Three hours following the return of the animals to their home cages, all animals were permitted to drink water for $20 \mathrm{~min}$ in the vivarium, the same as they had been permitted to do following each preexposure session and each context conditioning session.

Phase 4: Tests of blocking. The following day, all animals were placed in the drinking cages in the vivarium (hereafter referred to as the neutral test environment) for $20 \mathrm{~min}$, during which time they were free to ingest flavor $X$ from a drinking tube containing $40 \mathrm{cc}$ of the saccharin solution. This procedure was repeated each day, for 5 days, until the aversion to flavor $X$ was extinguished to the point at which there were no reliable differences in the amounts of flavor $\mathrm{X}$ ingested by the five groups.

Phase 5. Tests of conditioning of the contextual cues. The day following attainment of the extinction criterion in the neutral test environment, each of the five groups was tested for ingestion of flavor $\mathrm{X}$, the extinguished flavor $\mathrm{CS}$, in the cages of environment $C$. This procedure was repeated $20 \mathrm{~min}$ each day, for 4 days, until there was no evidence of differences among the five groups in the amounts of flavor $X$ ingested in the presence of the contextual cues.
Phase 6. Tests of conditioning of the novel exteroceptive cues. The following day, all animals were tested for ingestion of flavor $X$ in the presence of the novel exteroceptive cues of $B$, cues in the presence of which animals had not previously performed the ingestion response.

\section{Results and Discussion}

Figure 3 shows the mean amounts of flavor $X$ ingested by each of the five groups during each of the three phases of testing. The pattern of results revealed in Figure 3 was evaluated statistically by means of three separate 2 by 2 by $n$ analyses of variance based on the four experimental groups and the $\mathrm{n}$ tests conducted during each phase of testing. Three additional 2 by $n$ analyses of variance were used to compare the performance of the control group, Group $\mathrm{C} / \mathrm{CP} / \mathrm{CXP}$, with that of Group $\mathrm{C} / \mathrm{BCP} / \mathrm{CXP}$. When called for, the appropriate error term from the analysis of variance was used to compute the standard error of the mean for conducting individual $t$ tests.

Figure 3 contains four noteworthy findings. First, it is apparent that Groups $\mathrm{BCP} / \mathrm{BCXP}$ and $\mathrm{C} / \mathrm{BCP} /$ $\mathrm{BCXP}$ ingested significantly more of flavor $\mathrm{X}$ than did Groups BCP/CXP and C/BCP/CXP during the five tests conducted in the neutral test environment $[F(1,20)=7.26, p<.025]$. Thus, contrary to the Rudy et al. (1977) study, reinforcement of flavor X in compound with the novel exteroceptive cues (B) and the contextual cues $(\mathrm{C})$ resulted in greater blocking of the flavor aversion than did reinforcement of flavor $\mathrm{X}$ in compound with the contextual cues alone. This is also reflected in the more rapid rate at which animals in the former condition extinguished

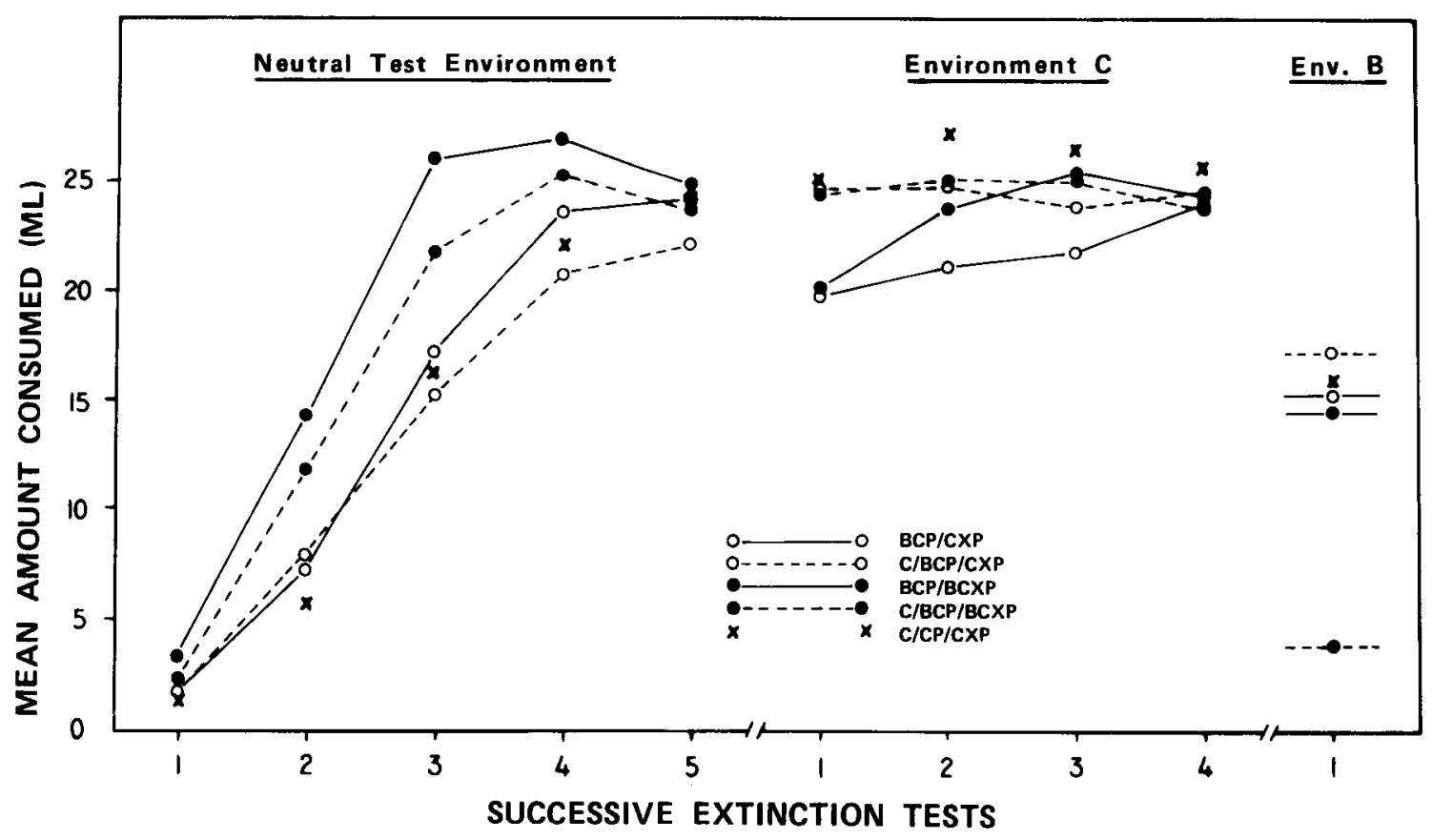

Figure 3. Mean amounts of flavor $\mathrm{CS}$ ingested in Experiment 3 by the four experimental groups and the control group during the five extinction tests conducted in the neutral test environment, the four extinction tests conducted in the presence of the contextual cues of environment $C$, and the single test conducted in the presence of the novel exteroceptive cues of environment $B$. 
the aversion over the five tests $[\mathrm{F}(4,80)=3.81, \mathrm{p}<.01$, for the Groups by Trials interaction].

The second finding of note in Figure 3 is that Group C/BCP/CXP ingested virtually the same amount of flavor $\mathrm{X}$ as did Group $\mathrm{C} / \mathrm{CP} / \mathrm{CXP}$, the control group, during the tests conducted in the neutral environment $(F<1)$. That the two groups did not differ in the rates at which they extinguished the aversion $(F<1)$ also suggests that the association between flavor $\mathrm{X}$ and the toxicosis was equally strong for both groups. Thus, it appears that reinforcement of the novel exteroceptive cues of $\mathrm{B}$ in compound with the contextual cues of $\mathrm{C}$ during the context conditioning phase did not nullify the effects of preexposing animals to the contextual cues.

The third finding of interest supports this contention. When tested in the neutral test environment, Groups $\mathrm{BCP} / \mathrm{BCXP}$ and $\mathrm{BCP} / \mathrm{CXP}$, the groups not preexposed to environment $C$, tended to ingest more of flavor $\mathrm{X}$ than did Groups $\mathrm{C} / \mathrm{BCP} / \mathrm{BCXP}$ and $\mathrm{C} / \mathrm{BCP} / \mathrm{CXP}$, respectively. However, when tested in the presence of the contextual cues of $C$ (i.e., following extinction of the aversion in the neutral test environment), Groups BCP/BCXP and BCP/CXP suppressed their ingestion of flavor $X$, whereas Groups C/BCP/BCXP and C/BCP/CXP did not. As a consequence, the rate at which the nonpreexposed animals (Groups BCP/BCXP and BCP/CXP) increased their ingestion of flavor $X$ over the four tests in $\mathrm{C}$ differed reliably from the rate at which the preexposed animals (Groups C/BCP/BCXP and $\mathrm{C} / \mathrm{BCP} / \mathrm{BXP})$ increased their ingestion $[\mathrm{F}(3,60)$ $=4.07, \mathrm{p}<.025$, for the Groups by Trials interaction]. Thus, even though each of the four groups of experimental animals was exposed to the novel exteroceptive cues of $B$ in sequential compound with the contextual cues of $\mathrm{C}$ during the context conditioning phase, the contextual cues appear to have acquired aversive conditioned properties only for those animals that had not been preexposed to the contextual cues. That Group C/BCP/CXP did not differ reliably from Group C/CP/CXP, the control group, either in terms of the overall amount of flavor $X$ that was ingested in $\mathrm{C}(\mathrm{F}<1)$ or in terms of the rate at which ingestion increased over the four tests $[F(3,30)=1.03$, $\mathrm{p}>.10$, again indicates that exposure to the novel exteroceptive stimulation during context conditioning did not cancel the effect of preexposing animals to the contextual cues.

This conclusion is also warranted by the fourth finding of interest. The results of the tests administered in the presence of novel exteroceptive cues of environment $B$ show that when each of the five groups was permitted to ingest flavor $\mathrm{X}$ in $\mathrm{B}$, ingestion of the flavor was far more suppressed in Group C/BCP/BCXP than it was in Group BCP/ BCXP $[t(20)=3.45, p<.01]$ or in Group C/BCP/ CXP $[\mathrm{t}(20)=4.28, \mathrm{p}<.01]$. This resulted in a significant interaction of the effects of preexposure or non- preexposure to $\mathrm{C}$, and exposure vs. nonexposure to $\mathrm{B}$ during flavor conditioning $[\mathrm{F}(1,20)=8.27, \mathrm{p}<.01]$. The difference between groups C/BCP/BCXP and $\mathrm{BCP} / \mathrm{BCXP}$ suggests that preexposure to the contextual cues of $C$ resulted in latent inhibition of the contextual cues in Group C/BCP/BCXP; as a consequence, the contextual cues of $C$ did not compete with the novel exteroceptive cues of $\mathrm{B}$ for association with toxicosis in Group C/BCP/BCXP during the context conditioning phase but did so in Group BCP/BCXP. This resulted in the novel exteroceptive cues of environment $\mathrm{B}$ acquiring more associative strength in Group $\mathrm{C} / \mathrm{BCP} / \mathrm{BCXP}$ than in Group BCP/BCXP, which, in turn, produced greater suppression of ingestion in Group C/BCP/ $\mathrm{BCXP}$ in $\mathrm{B}$. The fact that the first test conducted in the presence of the contextual cues of environment $C$ showed the contextual cues to cause a greater suppression of ingestion in Group $\mathrm{BCP} / \mathrm{BCXP}$ than in Group C/BCP/BCXP $[\mathrm{t}(20)=2.21, \mathrm{p}<.025]$ is consistent with this interpretation.

In summary, the pattern of results obtained in Experiment 3 confirms the findings of Experiments 1 and 2 and suggests, in contrast to the results obtained by Rudy et al. (1977), that: (1) preexposed contextual cues are not associated with toxicosis as readily as are nonpreexposed contextual cues, even if the contextual cues are reinforced in compound with novel exteroceptive cues; (2) preexposed contextual cues that are reinforced in compound with novel exteroceptive cues do not acquire more associative strength than preexposed contextual cues that are reinforced in the absence of novel exteroceptive cues; and (3) reinforcement of a novel flavor cue in sequential compound with novel exteroceptive cues and contextual cues that have been paired previously with poisoning results in a more attenuated flavor aversion than does reinforcement of the flavor cue in compound with the contextual cues alone.

\section{EXPERIMENT 4}

The purpose of Experiment 4 was to demonstrate further that the blocking effects observed in Experiments 1 and 3 were due to the conditioned properties of the novel exteroceptive cues (B) rather than to some nonassociative effect of the exteroceptive cues themselves. If the conditioned properties of the cues were responsible for the attenuated flavor aversions, then extinguishing the conditioned properties prior to reinforcing a flavor cue (X) in compound with the exteroceptive cues should result in a flavor aversion that is less attenuated than the aversion that develops in the absence of such extinction.

\section{Method}

The apparatus and subject population were the same as they were in Experiment 3, as were the procedural details, except for the following: None of the animals were exposed to the novel 
exteroceptive cues of environment B prior to Phase 1, the context conditioning phase (see Table 1). On each of the two context conditioning trials, animals from 'Jroup $B P / B(n=6)$ and Group BP/ rest $(n=6)$ were exposed to the novel exteroceptive cues of environment $B$ for $3 \mathrm{~min}$, at the end of which time they were injected with $100 \mathrm{mg} / \mathrm{kg}$ of $\mathrm{LiCl}$, returned to the boxes for $12 \mathrm{~min}$, and then returned to the vivarium.

Two hours following the second paired presentation of the novel exteroceptive cues (B) and the toxicosis (i.e., the second context conditioning trial), the animals in Group BP/B were returned to environment $B$ for $3 \mathrm{~min}$ and then removed from the boxes, administered a mock injection with a needleless syringe, and replaced in the boxes for $12 \mathrm{~min}$. During this same period of time, the animals in Group BP/rest were removed from their home cages in the vivarium, given the mock injection, and returned to their cages. Twelve minutes following the mock injection, the animals in Group BP/B were returned to the vivarium where they, as well as the animals in Group BP/rest, were placed in the drinking cages and permitted to drink water for $20 \mathrm{~min}$ before being returned to their home cages. This procedure was repeated on eight separate occasions and constituted Phase 2.

Upon removing the animals of Group BP/B from environment $B$ at the end of the first extinction session, the removable floor of each box was found to be littered with an exceptionally high number of boluses. Inasmuch as the floor of each box had been removed and cleaned following each of the context conditioning trials, an attempt was made to track the extinction process by recording the number of boluses found on the floor of each box after each extinction session. Thus, a group of animals that had previously experienced a $\mathrm{LiCl}$-induced poisoning in their home cages (in the context of another experiment) were given a second $100-\mathrm{mg} / \mathrm{kg}$ injection of $\mathrm{LiCl}$ in their home cages and $2 \mathrm{~h}$ later were placed in environment $B$ and given the mock injection, the same as had occurred for Group BP/B. These animals were used for the sole purpose of determining whether or not any observed reduction in the number of boluses excreted by Group BP/ $B$ might reflect the extinction of a conditioned response to the exteroceptive cues rather than the habituation of an unconditioned response to novelty.

Phase 3 of the experiment involved reinforcing a novel flavor cue, $\mathbf{X}$, in compound with the novel exteroceptive cues of environment $B$; this occurred the day following the eighth extinction session. Groups $\mathrm{BP} / \mathrm{B}$ and $\mathrm{BP} /$ rest were placed in the dark enclosed boxes of $B$ and permitted to ingest flavor $X$ (a .12\% saccharin solution, the novel flavor $\mathrm{CS}$ ) from drinking tubes that contained $20 \mathrm{cc}$ of the flavored solution. At the end of $7 \mathrm{~min}$, each animal received a $100-\mathrm{mg} / \mathrm{kg}$ injection of $\mathrm{LiCl}$ and was returned to $\mathrm{B}$ for $11 \mathrm{~min}$ before being returned to the vivarium. Three hours later, all animals were permitted to drink water for $20 \mathrm{~min}$ in the drinking cages in the vivarium.

Phase 4, which consisted of the tests for blocking of the flavor aversion, began the following day and involved exactly the same procedures as the tests for blocking conducted in the neutral environment in Experiment 3.

\section{Results and Discussion}

That the two context conditioning trials resulted in the formation of an association between the novel exteroceptive cues of B and the interoceptive US of toxicosis is evident in Figure 4. The figure shows that, following context conditioning, the reexposure of Groups BP/B to environment B resulted in the evocation of a conditioned gastrointestinal response (i.e., excretion of fecal material) that gradually diminished over the eight extinction sessions that intervened between context conditioning and flavor conditioning. Relative to the comparison group,

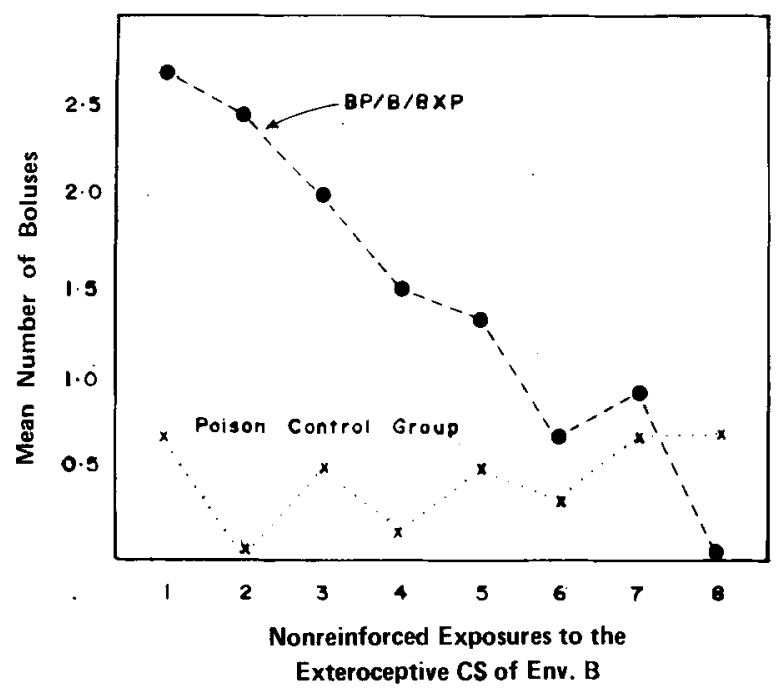

Figure 4. Mean number of boluses excreted by Group BP/B/BXP and the supplementary poison control group during the eight nonreinforced exposures to environment $B$ that intervened between context conditioning and flavor conditioning in Experiment 4.

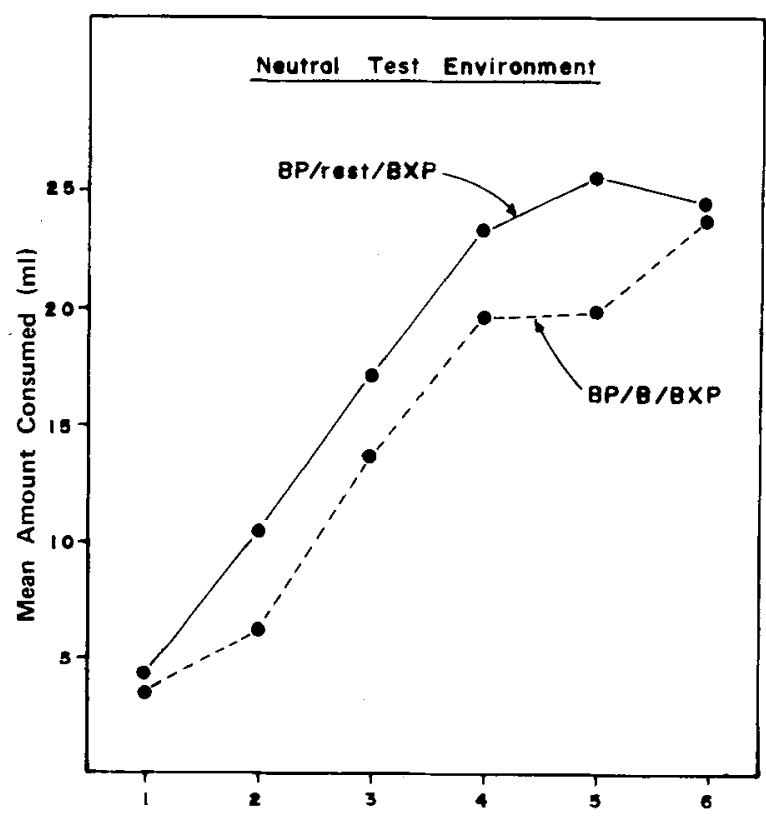

SUCCESSIVE EXTINCTION TESTS

Figure 5. Mean amounts of flavor $\mathrm{CS}$ ingested in Experiment 4 by Groups BP/B/BXP and BP/rest/BXP during the six extinction tests conducted in the neutral test environment.

Group BP/B excreted a significantly greater overall mean number of boluses during these sessions $[t(10)$ $=4.50, \mathrm{p}<.005]$. Thus it appears that the response observed in Group BP/B was, in fact, a conditioned response to the exteroceptive cues that had been paired with toxicosis rather than an unconditioned response to novelty.

Figure 5, which shows the mean amounts of flavor 
$\mathrm{X}$ ingested during the tests conducted in the neutral test environment, reveals that Group BP/rest ingested more of flavor X than did Group BP/B $[\mathrm{t}(10)=2.08$, $\mathrm{p}<.05]$. Thus, as predicted, the nonreinforced exposure of Group BP/B to the exteroceptive cues of B following context conditioning served to decrement the ability of the exteroceptive cues to subsequently block the formation of an association between the flavor CS and the toxicosis. It may be of interest to note that, during the preparation of this paper, Batson and Best (Note 1) reported a series of experiments in which the reinforcement of a flavor CS in compound with nonextinguished, as compared with extinguished, exteroceptive cues also resulted in greater blocking of a flavor aversion.

\section{GENERAL DISCUSSION}

The present experiments show that as few as two pairings of a novel exteroceptive CS and an interoceptive US can result in the exteroceptive CS's acquiring the ability to (1) elicit a conditioned gastrointestinal response that extinguishes as slowly as a conditioned aversion to a flavor CS, (2) block the development of an association between an interoceptive (flavor) $\mathrm{CS}$ and an interoceptive (illness) US, and (3) albeit to a lesser extent, suppress the ingestion of a neutral or a conditioned flavor stimulus.

That the "suppression-of-ingestion" measure provided weaker evidence of the conditioning of exteroceptive cues than did the former two measures may be significant. Inferences concerning the associability of exteroceptive cues and interoceptive reinforcers have been based primarily on the results of studies that failed to find little, if any, suppression of the ingestion response in the presence of extero-1 ceptive cues that had been paired with poison. In view of the strong evidence of conditioning that was obtained in the present experiments when conditioning of the exteroceptive cues was measured in terms of their ability to block the acquisition of a flavor aversion, or to elicit a conditioned gastrointestinal response, it is possible that the null effects observed in earlier studies (e.g., Garcia \& Koelling, 1966, 1967; Garcia, McGowan, Ervin, \& Koelling, 1968) were due to the particular response measure that was used, that is, a suppression-of-ingestion measure.

As noted earlier, Holland's (1977) research suggests that the form of the conditioned response that develops to a conditioned stimulus is determined, in part, by the qualitative characteristics of the CS. That different forms of conditioned responses may develop to exteroceptive cues and interoceptive cues that are paired with poison is suggested by the results of Experiment 4. When Group BP/B was reexposed to environment $B$ following the two context conditioning trials, the exteroceptive cues of $B$ readily evoked a conditioned gastrointestinal response that slowly extinguished with repeated nonreinforced exposures to $B$. The extinction of the conditioned properties of $B$ was further evidenced by the smaller amount of blocking that occurred in Group BP/B than in Group BP/rest when the flavor CS was reinforced in compound with the exteroceptive cues of $B$. In spite of the fact that the conditioned properties of B had been extinguished in Group BP/B but not in Group BP/rest prior to flavor conditioning, ingestion of the flavor CS in B on the flavor conditioning day was not found to differ reliably for the two groups (Group BP/B: mean $=8.50 \mathrm{ml}$; Group BP/ rest: mean $=6.02 \mathrm{ml})[\mathrm{t}(10)=1.53, \mathrm{p}>.10]$. The important point is that, while the reexposure of Group BP/B to environment B on the exteroceptive CS extinction trials elicited a strong conditioned gastrointestinal response, the reexposure of Group BP/ rest to the unextinguished cues of $B$ on the flavor conditioning trial did not cause a severe suppression of the ingestion response. Thus, it would appear that the form of the conditioned response that develops to exteroceptive cues that are paired with poison is very different from that which develops to an interoceptive cue that is paired with poison, at least if the poisoning occurs in the absence of consummatory behavior. However, the suppression of ingestion observed in environment B for Group BP in Experiment 1 and for Group C/BCP/BCXP in Experiment 3 also suggests that, once the ingestion response has occurred in the presence of exteroceptive cues and has been followed by poisoning, the exteroceptive cues may then elicit a conditioned response that is similar to that elicited by an interoceptive cue, that is, a conditioned response that serves to suppress the ingestion response.

In view of the complementary patterns of results that were obtained with the various measures of conditioning across the four experiments, it is reasonable to conclude that the attenuated flavor aversions obtained in Experiments 1, 3, and 4 represent blocking effects analogous to the blocking effects that have been observed in more conventional Pavlovian conditioning situations involving exteroceptive cues and exteroceptive reinforcers (Kamin, 1969; Rescorla \& Wagner, 1972). Moreover, because each group of animals in each experiment was exposed to the toxicosis equally often, the differences in the intensities of the aversions exhibited to the flavor CS during the tests for blocking cannot be attributed to differential nonassociative effects of the US (Braveman, 1975; Cannon, Berman, Baker, \& Atkinson, 1975).

In view of the discrepancy between the pattern of results observed in the Rudy et al. (1977) study and the pattern of results observed in the present study, it appears that additional research is needed to determine whether or not the attenuated flavor aversions obtained by Rudy et al. reflect the operation of the same processes that produced the blocking effects 
obtained in the present experiments. Unlike the effects observed by Rudy et al. (1977), the effects observed in the present experiments are directly analogous to those found in more conventional Pavlovian conditioning experiments. Moreover, during the preparation of this article, blocking effects similar to those reported here have also been reported by Willner (1978), who used procedures very much like those used in the present study, and by Batson and Best (Note 1), who used procedures very much like those used in the Rudy et al. (1977) study. Inasmuch as the results of the present research conform nicely to the outcomes that would be anticipated on the basis of Rescorla and Wagner's (1972) variable-reinforcement model of associative conditioning, or on the basis of the general stimulus-processing model recently proposed by Wagner (1976), the results provide further testimony of the generality of the laws of learning.

\section{REFERENCE NOTE}

1. Batson, J. D., \& Best, P. J. Blocking of taste-aversion learning by environmental cues; The illness pre-exposure effect as an associative process. Paper presented at the annual meeting of the Eastern Psychological Association, Washington, D.C., April 1978.

\section{REFERENCES}

Best, P. J., Best, M. R., \& Mickley, G. A. Conditioned aversion to distinct environmental stimuli resulting from gastrointestinal distress. Journal of Comparative and Physiological Psychology, 1973, 85, 250-257.

Bitterman, M. E. The comparative analysis of learning. Science, 1975, 188, 699-709.

Bitterman, M. E. Flavor aversion studies: Technical comment. Science, 1976, 192, 266-267.

Blanchard, R., \& Honig, W. K. Surprise value of food determines its effectiveness as a reinforcer. Journal of Experimental Psychology: Animal Behavior Processes, 1976, 2, 67-74.

Braveman, N. S. Formation of taste aversions in rats following prior exposure to sickness. Learning and Motivation, 1975, 6, 512-534.

Cannon, D. S., Berman, R. F., Baker, T. B., \& Atkinson, C. A. Effect of preconditioning unconditioned stimulus experience on learned taste aversions. Journal of Experimental Psychology: Animal Behavior Processes, 1975, 1, 270-284.

Garcia, J., \& Koelling, R. A. Relation of cue to consequence in avoidance learning. Psychonomic Science, 1966, 4, 123-124.

Garcia, J., \& Koelling, R. A. A comparison of aversions induced by $\mathrm{X}$-rays, toxins, and drugs in the rat. Radiation Research Supplement, 1967, 7, 439-450.

Garcia, J., McGowan, B. K., Ervin, F. R., \& Koelling, R. A. Cues-their relative effectiveness as a function of the reinforcer. Science, 1968, 160, 794-795.

Holland, P. C. Conditioned stimulus as a determinant of the form of the Pavlovian conditioned response. Journal of Experimental Psychology: Animal Behavior Processes, 1977, 3, 77-104.
Holland, P. C., \& Resconla, R. A. The effect of two ways of devaluing the unconditioned stimulus after first- and secondorder appetitive conditioning. Journal of Experimental Psychology: Animal Behavior Processes, 1975, 1, 355-363. (a)

Holland, P. C., \& Rescorla, R. A. Second-order conditioning with food unconditioned stimulus. Journal of Comparative and Physiological Psychology, 1975, 88, 459-467. (b)

Kamin, L. J. Selective association and conditioning. In N. J. MacKintosh \& W. K. Honig (Eds.), Fundamental issues in associative learning. Halifax: Dalhousie University Press, 1969.

Krane, R. V., \& Wagner, A. R. Taste-aversion learning with a delayed shock US: Implications for the "generality of the laws of learning." Journal of Comparative and Physiological Psychology, 1975, 88, 882-889.

Lubow, R. E. Latent inhibition. Psychological Bulletin, 1973, 79, 398-407.

Mitchell, D., Kirschbaum, E. H., \& Perry, R. L. Effects of neophobia and habituation on the poison-induced avoidance of exteroceptive stimuli in the rat. Journal of Experimental Psychology: Animal Behavior Processes, 1975, 104, 47-55.

Rescorla, R. A. Second-order conditioning: Implications for theories of learning. In F. J. McGuigan \& D. B. Lumsden (Eds.), Contemporary approaches to conditioning and learning. Washington, D.C: Winston \& Sons, 1973.

Rescorla, R. A., \& Furrow, D. R. Stimulus similarity as a determinant of Pavlovian conditioning. Journal of Experimental Psychology: Animal Behavior Processes, 1977, 3, 203-215.

Rescorla, R. A., \& W AGner, A. R. A theory of Pavlovian conditioning: Variations in the effectiveness of reinforcement and nonreinforcement. In A. H. Black \& W. F. Prokasy (Eds.), Classical conditioning II: Current theory and research. New York: Appleton-Century-Crofts, 1972.

REvUsKy, S., \& GARCIA, J. Learned associations over long delays. In G. Bower \& J. T. Spence (Eds.,), Psychology of learning and motivation. New York: Academic Press, 1970.

Revusky, S., \& Parker, L. A. Aversions to unflavored water and cup drinking produced by delayed sickness. Journal of Experimental Psychology: Animal Behavior Processes, 1976, 2, 342-353.

Rozın, P. Specific aversions and neophobia resulting from vitamin deficiency or poisoning in half-wild and domestic rats. Journal of Comparative and Physiological Psychology, 1968, 66, 82-88.

Rozin, P. Central vs. peripheral mediation of learning with long CS-US intervals in the feeding system. Journal of Comparative and Physiological Psychology, 1969, 67, 421-429.

Rudy, J. A., Iwens, J., \& Best, P. J. Pairing novel exteroceptive cues and illness reduces illness-induced taste-aversions. Journal of Experimental Psychology: Animal Behavior Processes, 1977, 3, 14-25.

Wagner, A. R. Priming in STM: An information processing mechanism for self-generated or retrieval-generated depression in performance. In T. J. Tighe \& R. N. Leaton (Eds.), Habituation: Perspectives from child development, animal behavior, and neurophysiology. Hillsdale, N.J: Erlbaum, 1976.

Willner, J. A. Blocking of a taste aversion by prior pairings of exteroceptive stimuli with illness. Learning and Motivation, 1978, 9, 125-140.

(Received for publication April 6, 1979; revision accepted June 13,1980 .) 